\title{
Molecular characterisation of multidrug-resistant Pseudomonas aeruginosa from a private hospital in Durban, South Africa
}

\author{
Cosmos B. Adjei ${ }^{\mathrm{a}}$, Usha Govinden ${ }^{\mathrm{a}}$, Krishnee Moodley ${ }^{\mathrm{b}}$ and Sabiha Y. Essack ${ }^{\mathrm{a} *}$ (iD \\ ${ }^{a}$ Antimicrobial Research Unit, University of KwaZulu-Natal, Westville, Durban, South Africa \\ 'Lancet Laboratories, Durban, South Africa \\ *Corresponding author, email: essacks@ukzn.ac.za
}

Background: Multi-drug resistant Pseudomonas aeruginosa pose a clinical challenge globally. This study delineated the molecular mechanisms of resistance to $\beta$-lactam antibiotics in multidrug-resistant $P$. aeruginosa isolated from a single private hospital in Durban, South Africa and ascertained clonality with regard to the isolates carrying $\beta$-lactamase genes.

Methods: Seventeen P. aeruginosa isolates recovered from sputum, urine, catheter tips, pus swabs, nasal swabs and endotracheal aspirates underwent MIC determination, and phenotypic screening using the Double Disk Synergy Test (DDST) and Modified Hodge Test (MHT) to identify putative extended-spectrum $\beta$-lactamases (ESBLs), metallo- $\beta$-lactamases and other carbapenemases. Selected $\beta$-lactamase encoding genes were genotypically confirmed by PCR and sequencing. REP-PCR was conducted to determine the clonal relatedness of the 11 isolates carrying $\beta$-lactamase genes.

Results: Sixteen isolates (94\%) were resistant to aztreonam and piperacillin, 15 isolates (88\%) were resistant to imipenem and ticarcillin, 14 (82\%) were resistant to meropenem, and 13 isolates (76\%) were resistant to ceftazidime and piperacillin/tazobactam. Resistance to ciprofloxacin and amikacin were $82 \%$ and $29 \%$ respectively. Of the 17 isolates tested, GES-2, VIM-2 and OXA-21 were present in $10(59 \%)$ four $(24 \%)$ and one $(6 \%)$ of the isolates respectively. Three of the isolates harboured both GES-2 and VIM-2 and one isolate harboured OXA-21 and VIM-2. REP-PCR revealed seven clusters with clusters A and F having two (18\%) and four (36\%) isolates respectively, while the remaining five isolates were unrelated.

Conclusion: GES-2 and VIM-2 enzymes were predominantly responsible for carbapenemase resistance. Clones A and F intimated patient-to-patient spread within the ICU and surgical ICU. This apparent dissemination as well as the multi-drug resistance observed points to sub-optimal infection prevention and control and dwindling antibiotic treatment options for $P$. aeruginosa respectively in this institution.

Keywords: multi-drug resistance, Pseudomonas aeruginosa, GES, VIM

\section{Introduction}

Pseudomonas aeruginosa is a well-known opportunistic organism that is commonly implicated in nosocomial infections. ${ }^{1} P$. aeruginosa has intrinsic resistance to a number of antimicrobial agents and is also capable of acquiring resistance genes from other bacteria via mobile genetic elements. As a result, it is coresistant to several antibiotics such as the $\beta$-lactams, aminoglycosides and fluoroquinolones. ${ }^{2}$ Treatment options for infections caused by multi-drug resistant (MDR) P. aeruginosa are limited, making it one of the most challenging pathogens for antimicrobial chemotherapy. Patients in the intensive care, burn and surgery units are particularly prone to infection by MDR $P$. aeruginosa, thus contributing to high mortality rates as well as high treatment cost. ${ }^{3}$

In $P$. aeruginosa, metallo- $\beta$-lactamases (MBLs) such as VIM, IMP, SPM, AIM and GIM present the leading acquired resistance mechanism to carbapenems. ${ }^{4}$ Although VIM and IMP variants have been reported globally, SPM, AIM and GIM are confined to particular geographical locations. ${ }^{5}$ The evolution and/or dissemination of ESBLs, namely CTX-M, SHV, TEM, VEB, PER, GES and OXA-type, in $P$. aeruginosa is a further clinical challenge specifically as these enzymes are known to be plasmid mediated and undergo horizontal gene transfer. ${ }^{6}$

We delineated the molecular mechanisms of resistance to $\beta$-lactam antibiotics in multidrug-resistant $P$. aeruginosa isolated from a case series in a single private hospital in Durban, South
Africa and ascertained clonality in respect of isolates carrying $\beta$-lactamase genes.

\section{Methods}

\section{Ethical consideration}

This study was ethically approved by the Biomedical Research Ethics committee of the University of KwaZulu-Natal (BE 224/15).

\section{Sample}

Seventeen $P$. aeruginosa isolates that were consecutively collected from different patients admitted to a private hospital in Durban, from August to October 2013 constituted the sample. Single isolates were recovered from sputum, urine, catheter tips, pus swabs, nasal swabs and endotracheal aspirate from patients in the outpatients department $(n=1)$, medical ward $(n=1)$, theatre $(n=1)$, intensive care unit $(n=9)$ and surgical intensive care unit $(n=5)$ of a single private hospital.

An MDR P. aeruginosa isolate was defined as an isolate that was resistant to at three or more classes of antibiotics.?

\section{Antibiotic susceptibility testing}

Minimum inhibitory concentrations (MIC) of piperacillin, ticarcillin, piperacillin/tazobactam, ceftazidime, aztreonam, imipenem, meropenem, amikacin and ciprofloxacin were determined by agar dilution method according to CLSI recommendations. ${ }^{8} P$. aeruginosa ATCC 27853 was used as the control. 


\section{Phenotypic screening for MBLs and ESBLs}

All isolates were screened for MBLs using the imipenem-EDTA double disk synergy test (DDST) as described by Bashir et al. ${ }^{9}$ An increase in the zone size of at least $7 \mathrm{~mm}$ around the imipenemEDTA disc was recorded as an MBL-positive isolate. ${ }^{9}$

For the phenotypic detection of carbapenemases, the Modified Hodge's test (MHT) was performed on MHA using a $10 \mu \mathrm{g}$ disk of meropenem according to CLSI guidelines. ${ }^{8}$

All the isolates were evaluated for ESBL production using a Mueller-Hinton agar (MHA) plate and ceftazidime $(30 \mu \mathrm{g})$ and ceftazidime/clavulanic acid $(30 \mu \mathrm{g} / 10 \mu \mathrm{g})$ discs. The observation of $a \geq 5 \mathrm{~mm}$ increase in the zone diameter for the ceftazidime combination with clavulanic acid, versus its zone diameter when tested alone, confirmed the presence of ESBL production by the organism. ${ }^{8}$

\section{Polymerase chain reaction}

DNA was extracted from overnight bacterial cultures with high pure DNA isolation kits (Roche molecular diagnostics, Roche Molecular Systems, Pleasanton, CA, USA) as per manufacturers' guidelines. DNA amplification was performed in a $\mathrm{T} 100^{\mathrm{TM}}$ Thermal cycler (Bio-Rad Laboratories, Johannesburg, South Africa) in a final volume of $25 \mu \mathrm{l}$, containing $1.25 \mu \mathrm{l}$ (100 pmol) of each primer, $1 \mu \mathrm{l}$ template DNA, and $12.5 \mu \mathrm{l}$ of master mix (Thermo Fisher Scientific, Waltham, MA, USA). PCR for bla- ${ }_{{ }_{\mathrm{TEM}}}$ bla- ${ }_{\mathrm{SHV}^{\prime}}$ and bla- ${ }_{\text {CTX-M }}$ was carried out as previously described by Jemima et al. ${ }^{10}$ PCR amplification with some modifications in the annealing temperatures for $b l a_{\mathrm{OXA}-2^{\prime}}, b l a_{\mathrm{OXA}-10^{\prime}}, b l a_{\mathrm{OXA}_{-48^{\prime}}} b l a_{\mathrm{VIM}^{\prime}} b l a_{\mathrm{NDM}^{\prime}} b l a_{\mathrm{GES}^{\prime}}$ $b / a_{\mathrm{VEB}}, b / a_{\mathrm{IMP}}$ and $b / a_{\mathrm{PER}}$ was performed as previously described by
Shacheraghi et al. ${ }^{11}$ and Poirel et al. ${ }^{12}$ The annealing temperatures and primers are described in Table 1. PCR products were visualised by electrophoresis in $1.5 \%$ agarose gel for $40 \mathrm{~min}$ at $120 \mathrm{~V}$ then sequenced using Sanger Dideoxy sequencing technology (Inqaba Biotech, Pretoria, South Africa). Analysis of the sequences was done using BLAST 2.0 (Basic Local Alignment Search Tool) software available on the National Center for Biotechnology Information website (https://www.ncbi.nhlm.nih. gov/blast/BLAST.cgi) and BioEdit (http://www.mbio.ncsu.edu/ BioEdit/bioedit.html). Positive controls (P. aeruginosa ATCC 27853 and E. coli ATCC 35218) were run along with the tested samples.

\section{Repetitive extragenic palindromic-polymerase chain reaction ( $R E P-P C R)$}

REP-PCR amplification was carried out on isolates carrying $\beta$-lactamase genes according to Dawson et al. ${ }^{13}$ in a total reaction volume of $50 \mu$ containing $10 \mathrm{mM}$ dNTP, $25 \mathrm{mM} \mathrm{MgCl} 2,10 \mathrm{pmol}$ of primer (Table 1), $2 \mu$ lof the genomic template DNA, and $5 \mathrm{U}$ of Taq DNA polymerase (Thermo Fisher Scientific, USA).

Initial denaturation was at $94^{\circ} \mathrm{C}$ for $10 \mathrm{~min}$ followed by 30 cycles of PCR consisting of denaturation at $94^{\circ} \mathrm{C}$ for $1 \mathrm{~min}$, annealing at $45^{\circ} \mathrm{C}$ for $1 \mathrm{~min}$, and extension at $72^{\circ} \mathrm{C}$ for $2 \mathrm{~min}$, with the final extension of $16 \mathrm{~min}$. The PCR product was run on a $2 \%$ agarose gel in TBE buffer. Images were captured with the Bio-Rad ChemiDoc $^{\mathrm{TM} M}$ MP System (Bio-Rad Laboratories, Johannesburg, South Africa) and analysed with the BioNumerics software (Applied Maths, Sint-Martens-Latem, Belgium). The Dice coefficient and unweighted-pair group method was used to generate the dendrogram with $1.0 \%$ tolerance and $0 \%$ optimisation values. ${ }^{14}$

Table 1: Antimicrobial susceptibility and genotypic profile of $P$. aeruginosa

\begin{tabular}{|c|c|c|c|c|c|c|c|c|c|c|c|c|c|c|c|c|}
\hline \multirow[t]{2}{*}{ Isolate } & \multirow{2}{*}{$\begin{array}{l}\text { Specimen } \\
\text { type }\end{array}$} & \multirow[t]{2}{*}{ Unit } & \multicolumn{8}{|c|}{ MIC ( $\mu \mathrm{g} / \mathrm{ml})$} & \multirow[t]{2}{*}{ TZP } & \multirow{2}{*}{$\begin{array}{c}\text { DDST } \\
\text { (MBLs) }\end{array}$} & \multirow{2}{*}{$\begin{array}{c}\text { DDST } \\
\text { (ESBLs) }\end{array}$} & \multirow[t]{2}{*}{ MHT } & \multirow{2}{*}{$\begin{array}{l}\text { REP-PCR } \\
\text { (clusters) }\end{array}$} & \multirow{2}{*}{$\begin{array}{c}\text { Genes } \\
\text { identified }\end{array}$} \\
\hline & & & ATM & AK & CAZ & CIP & IMP & PIP & MEM & TIC & & & & & & \\
\hline pa1 & Wound & SC-ICU & 128 & 16 & 32 & 64 & 32 & $\geq 512$ & 32 & $\geq 512$ & 32 & + & + & + & $F$ & GES-2 \\
\hline pa2 & Sputum & SC-ICU & 64 & 1 & 8 & $\leq 0.25$ & 2 & 16 & 2 & 32 & 16 & - & - & - & - & - \\
\hline pa4 & CVP tip & SC-ICU & 256 & 8 & 4 & 32 & $\geq 64$ & $\geq 512$ & 128 & $\geq 512$ & 256 & + & - & - & - & - \\
\hline pa6 & ETA & ICU & 128 & 16 & 32 & 32 & $\geq 64$ & $\geq 512$ & 64 & $\geq 512$ & 64 & + & + & + & $A$ & GES-2 \\
\hline pa7 & Sputum & ICU & 128 & 64 & 64 & 64 & 32 & $\geq 512$ & 8 & $\geq 512$ & 32 & + & + & + & $F$ & GES-2 \\
\hline pa8 & Pus swab & SC-ICU & 2 & $\leq 0.25$ & 32 & $\leq 0.25$ & 2 & 128 & $\leq 0.25$ & 32 & 16 & + & - & - & - & - \\
\hline pa12 & Sputum & ICU & 256 & 32 & 4 & 64 & 32 & $\geq 512$ & 4 & $\geq 512$ & 128 & + & - & - & - & - \\
\hline pa17 & Sputum & SC-ICU & 64 & 16 & 64 & 8 & $\geq 64$ & 256 & 8 & $\geq 512$ & 128 & + & + & + & $F$ & GES-2 \\
\hline pa18 & Sputum & ICU & 64 & $\leq 0.25$ & 64 & 16 & $\geq 64$ & $\geq 512$ & 64 & $\geq 512$ & 32 & + & + & + & $\mathrm{F}$ & GES-2 \\
\hline pa19 & Sputum & OPD & 64 & 16 & 64 & 8 & $\geq 64$ & 256 & 256 & $\geq 512$ & 128 & + & + & + & $E$ & $\begin{array}{l}\text { GES-2, } \\
\text { VIM-2 }\end{array}$ \\
\hline pa21 & Sputum & $\begin{array}{l}\text { Med. } \\
\text { ward }\end{array}$ & 256 & 32 & 32 & 64 & $\geq 64$ & $\geq 512$ & 512 & $\geq 512$ & 256 & + & + & + & D & $\begin{array}{l}\text { GES-2, } \\
\text { VIM-2 }\end{array}$ \\
\hline pa22 & Urine & Theatre & 16 & $\leq 0.25$ & 8 & $\leq 0.25$ & 32 & $\geq 512$ & 4 & 256 & 8 & + & - & - & - & - \\
\hline pa23 & Sputum & ICU & 256 & 32 & 32 & 64 & $\geq 64$ & $\geq 512$ & 512 & $\geq 512$ & 128 & + & + & + & B & GES-2 \\
\hline pa26 & CVP tip & ICU & 32 & 1 & 64 & 8 & $\geq 64$ & 128 & 512 & $\geq 512$ & 128 & + & + & + & A & $\begin{array}{l}\text { GES-2, } \\
\text { VIM-2 }\end{array}$ \\
\hline pa28 & Sputum & ICU & 128 & 4 & 128 & 32 & $\geq 64$ & $\geq 512$ & 64 & 512 & 128 & + & + & + & G & $\begin{array}{l}\text { VIM-2, } \\
\text { OXA-21 }\end{array}$ \\
\hline pa30 & Sputum & ICU & 128 & 16 & 64 & 16 & $\geq 64$ & $\geq 512$ & 128 & $\geq 512$ & 256 & + & + & + & C & GES-2 \\
\hline pa31 & Nasal swab & ICU & 256 & 4 & 32 & 32 & $\geq 64$ & $\geq 512$ & 64 & $\geq 512$ & 128 & + & + & - & - & - \\
\hline
\end{tabular}

ATM: aztreonam; AK: amikacin; CAZ: ceftazidime; CIP: ciprofloxacin; IMP: imipenem; PIP: piperacillin; MEM: meropenem; TIC: ticarcillin TZP; piperacillin/tazobactam; DDST: double disc synergy test; ESBL: extended spectrum $\beta$-lactamase; MBL: metallo $\beta$-lactamase; MHT: Modified Hodge Test; ICU: intensive care unit; ETA: endotracheal aspirate; SC-ICU: surgical intensive care unit; OPD: outpatients department; Med. ward: medical ward; CVP: central venous catheter. 


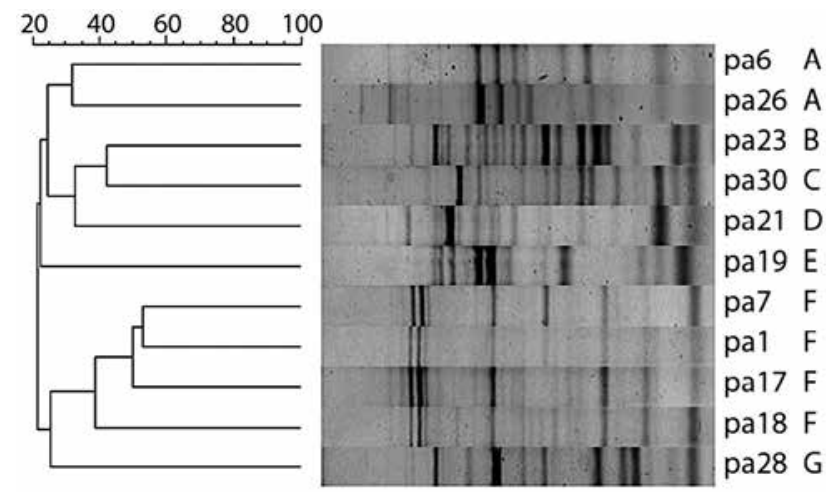

Figure 1: REP-PCR analysis. Dendrogram and computer-generated image of REP-PCR banding patterns of isolates harbouring ESBL and MBL genes.

\section{Results}

Sixteen isolates (94\%) were resistant to aztreonam and piperacillin, 15 (88\%) were resistant to imipenem and ticarcillin, $14(82 \%)$ were resistant to meropenem, and 13 (76\%) were resistant to ceftazidime and piperacillin/tazobactam. Resistance to ciprofloxacin and amikacin were $82 \%$ and $29 \%$ respectively (see Table 1). The double disc synergy test (DDST) for MBLs and ESBLs was positive in $15(88 \%)$ and $13(76 \%)$ respectively of the $P$. aeruginosa isolates while MHT was positive in 11 (65\%) (see Table 1). PCR identified the GES-2 gene in 10 isolates (59\%), the VIM-2 gene in four isolates (24\%) and OXA-21 in one (6\%) isolate. Three of the isolates harboured both GES-2 and VIM-2 and one isolate harboured OXA-21 and VIM-2 as shown in Table 1.

\section{REP-PCR clonality}

REP- PCR was conducted to determine genetic similarity of the $\beta$-lactamase-producing isolates harbouring the resistance genes among 11 isolates. Seven clusters (A-G) were revealed, with two (18\%) and four (36\%) of the P. aeruginosa isolates belonging to clusters $A$ and $F$ respectively whereas the remaining were unique and belonged to five unrelated clusters (clusters B, C, D, E, and G) (Figure 1).

\section{Discussion}

This study aimed to delineate the molecular mechanism of resistance to $\beta$-lactam antibiotics in multidrug-resistant $P$. aeruginosa isolated from a private hospital. It further ascertained clonality to inform infection prevention and control measures.

Infections caused by MDR $P$. aeruginosa are commonly reported in ICU patients worldwide. ${ }^{15}$ Studies have indicated that the ICUs are the epicentre of pathogens ${ }^{16}$ and the highly resistant nature of this pathogen poses a major treatment challenge particularly when carbapenems, which used to be the treatment of choice for MDR $P$. aeruginosa infection, have been rendered inefficient due to production of carbapenemases. ${ }^{17}$

In a study conducted at the Charlotte Maxeke Johannesburg Academic Hospital (CMJAH) to determine the patterns of infection and bacterial resistance in critically ill poly-trauma patients admitted to ICU, it was shown that $30.1 \%$ of MDR $P$. aeruginosa was the commonest pathogen. ${ }^{18}$ In another South African hospital, eight $P$. aeruginosa clinical strains involved in nosocomial outbreak were all isolated from the ICU. ${ }^{19}$ Pitout et al. reported that majority of isolates recovered in a large tertiarycare centre in Nairobi, Kenya were P. aeruginosa isolates (33/57, $58 \%$ ) and they originated from the ICU. ${ }^{20}$ In a study on an outbreak of multidrug-resistant $P$. aeruginosa (MDRPA) infections in a Greece university hospital, it was shown that after phenotypic and genotypic analysis of 240 isolates mainly in ICU, 152 patients were colonised or infected with MDRPA. ${ }^{21} \mathrm{~A}$ study by Harris et al. to investigate the prevalence of $P$. aeruginosa colonisation on ICU admission, revealed that of the 1840 patients, 213 (11.6\%) were colonised with $P$. aeruginosa on ICU admission. ${ }^{22}$ In another study on frequency of multi-drug resistant (MDR) $P$. aeruginosa in intensive care unit (ICU)-acquired pneumonia (ICUAP), $P$. aeruginosa was the most frequent aetiology of ICUAP $(64,29 \%) .^{23}$ These are in line with our studies where $82 \%$ of the isolates were recovered from the ICUs.

GES-2 and OXA-21, and VIM-2 were the predominant ESBLs and MBLs identified.

GES-2 hydrolyses extended spectrum cephalosporins and imipenem, to a minor extent ${ }^{24}$ explaining the resistance to ceftazidime but the high resistance to the carbapenems may be attributed to resistance mechanisms other than $\beta$-lactamases such as impermeability and/or efflux. ${ }^{25}$

VIM-2, a derivative of VIM-1, possesses the widest substrate for hydrolysing several antimicrobial agents such as carbapenems, cephalosporins and penicillin. All four isolates harbouring VIM-2 enzymes were resistant to imipenem, meropenem, ceftazidime and ticarcillin (see Table 1).

Oxacillinases hydrolyse meropenem and imipenem poorly, and do not hydrolyse extended-spectrum cephalosporins and aztreonam. ${ }^{24}$ However, in this study OXA-21 was detected together with VIM-2 and showed resistance to all classes of antibiotics tested (see Table 1) with the exception of aminoglycosides.

GES-2 and OXA-21 were identified in 10 (59\%) and one (6\%) isolates respectively. Several GES-type ESBLs have been identified and some exhibit the properties of carbapenemases. They have been found in a number of geographical locations including French Guiana, Japan, Greece, France and South Africa. ${ }^{25}$ GEStype ESBLs in $P$. aeruginosa are not considered as primary $\beta$-lactamases, but their acquisition is due to antimicrobial pressure. ${ }^{6}$ The GES-2-producing isolates in this study were all resistant to carbapenems and third-generation cephalosporins. They were all positive for MHT, indicating the production of carbapenemases genes. GES-2 was described originally in multidrug-resistant $P$. aeruginosa isolates collected from a South African teaching hospital, were associated with a nosocomial outbreak and displayed expanded hydrolysis to carbapenems. ${ }^{19}$

The detection of class $B \mathrm{MBL}, \mathrm{VIM}-2$ in these isolates is in agreement with results from other studies indicating that VIM-2 is amongst the most widespread MBLs associated with P. aeruginosa. ${ }^{26}$ VIM-2 has also been implicated in outbreaks of nosocomial infections as a result of MBL-producing $P$. aeruginos $a .{ }^{26}$ Three other isolates were found to harbour VIM-2 and GES-2. The simultaneous production of ESBLs and MBLs by the same isolate is known to further enhance resistance. ${ }^{27}$

Many of the Ambler class D ESBLs have been found and often described in $P$. aeruginosa. Nearly all class $D \beta$-lactamases produced by $P$. aeruginos $a$ are capable of hydrolysing extendedspectrum cephalosporins, with OXA-1 and OXA-2 derivatives being the commonest OXA type detected. ${ }^{28}$ In this study OXA-21, a derivative of OXA-2, was identified. OXA-21 was identified 
together with VIM-2 in the same isolate from an ICU in a patient's sputum and found to be resistant to all antibiotics with the exception of amikacin, and the only isolate that was resistant to colistin. Moreover, whereas GES-2 and VIM-2 were the dominant ESBL and MBL respectively, ESBLs such as SHV, TEM and PER, as well as MBLs such as NDM and IMP, were absent in these isolates.

The clonal relationship between $11 P$. aeruginosa isolates revealed that two (18\%) and four (36\%) belonged to clusters $A$ and $F$ respectively while the remaining isolate showed unique patterns. With exception of one isolate, all isolates in clusters $A$ and $F$ harboured GES-2, intimating dissemination within the ICU and surgical ICU.

\section{Conclusion}

The $P$. aeruginosa strains in this study were multidrug resistant expressing both ESBLs and MBLs. VIM-2 was the only MBL found whereas GES-2 was the most prevalent ESBL detected. The detection of GES-2 with VIM-2 in three isolates and the coexistence of VIM-2 and OXA- 21 in one isolate depict the extraordinary ability of $P$. aeruginosa to acquire multiple resistance mechanisms. The clonal relatedness and the multidrug resistance observed points to sub-optimal infection prevention and control and dwindling antibiotic treatment options for $P$. aeruginos a respectively in this institution. Treatment of $P$. aeruginosa infections in the hospital setting must therefore be guided by local antimicrobial susceptibility patterns.

Funding - This work was supported by the National Research Foundation [grant number 85595].

\section{ORCID}

Sabiha Y. Essack (D) http://orcid.org/0000-0003-3357-2761

\section{Supplemental data}

Supplemental data for this article can be accessed at https://doi. org/10.1080/23120053.2017.1382090.

\section{References}

1. Slama TG. Gram-negative antibiotic resistance: there is a price to pay. Crit Care. 2008;12(4): S4. https://doi.org/10.1186/cc6820

2. DundarD,OtkunM.In-VitroEfficacy ofSynergistic Antibiotic Combinations in Multidrug Resistant Pseudomonas Aeruginosa Strains. Yonsei Med J. 2010;51(1): 111-116. https://doi.org/10.3349/ymj.2010.51.1.111

3. Giamarellos-Bourboulis EJ, Papadimitriou E. Galanakis N, al e. Multidrug resistance to antimicrobials as a predominant factor influencing patient survival. Int J Antimicrob Agents. 2006;27(6): 476-81. https://doi.org/10.1016/j.ijantimicag.2005.12.013

4. Zhao $\mathrm{WH}, \mathrm{Hu} Z \mathrm{ZQ}$. $\beta$-Lactamases identified in clinical isolates of Pseudomonas aeruginosa. Crit Rev Microbiol. 2010;36: 245-58. https://doi.org/10.3109/1040841X.2010.481763

5. Ellington MJ, Kistler J, Livermore D, Woodford N. Multiplex PCR for rapid detection of genes encoding acquired metallo- beta lactamases. J Antimicrob Chemother. 2007;59(2): 321-2.

6. Labuschagne J, Weldhagen G, Ehlers M, Dove M. Emergence of class 1integron-associated GES-5 and GES-5-like extended-spectrum B-lactamases in clinical isolates of Pseudomonas aeruginosa in South Africa. Int J Antimicrob Agents 2008;31: 527-30. https://doi. org/10.1016/j.ijantimicag.2008.01.020

7. Kallen AJ, Hidron Al, Patel J, Srinivasan A. Multidrug Resistance among Gram-Negative Pathogens That Caused Healthcare-Associated Infections Reported to the National Healthcare Safety Network, 2006-2008. Inf Cont Hosp Epidemiol. 2010;31(5): 528-31. https://doi. org/10.1086/651677

8. CLSI. Performance standards for antimicrobial susceptibility testing; twenty-fourth informational supplement document M100-S24. Clin Lab Stand Ins. 2014;34 (1):58-60.

9. Bashir D, Thokar MA, Fomda BA, Bash G, Zahoor DSA. A. and Toboli, A.S. Detection of metallobeta- lactamases (MBL) producing
Pseudomonas aeruginosa at a tertiary care hospital in Kashmir.. Afr J Microbiol 2011;5: 164-72.

10. Jemima SA, Verghese S. Multiplex PCR for bla CTX-M \& bla SHV in the extended spectrum $\beta$ - lactamase (ESBL) producing Gram-negative isolates. Indian J Med Res. 2008;128: 313-7.

11. Shacheraghi $F$, Shakibaie MR, Noveiri H. Molecular Identification of ESBL genes blaGES-1, blaVEB-1, blaCTX-M blaOXA-1, blaOXA-4, blaOXA-10 and blaPER-1 in Pseudomonas aeruginosa strains isolated from burn patients by PCR, RFLP and sequencing techniques. Int J Biol life Sci. 2010;3(6): 138-42.

12. Poirel L, WalshTR, CuvillierV, Nordmann P. MultiplexPCRfor detection of acquired carbapenemase genes. Diagn Microbiol Infect Dis. 2011;70(1): 119-23. https://doi.org/10.1016/j.diagmicrobio.2010.12.002

13. Dawson S, Fry JC, Dancer BN. A comparative evaluation of five typing techniques for determining the diversity of fluorescent pseudomonads. J Microbiol Meth. 2002;50: 9-22. https://doi. org/10.1016/S0167-7012(02)00003-9

14. Bou G, Cervero G, Dominguez A, Quereda C. PCR-based DNA fingerprinting (REP-PCR, AP-PCR) and pulsed-field gel electrophoresis characterization of a nosocomial outbreak caused by imipenem- and meropenem-resistant Acinetobacter baumannii. Clin Microbiol Infect. 2000;6: 635-43. https://doi.org/10.1046/j.1469-0691.2000.00181.x

15. Kollef $M$, Micek S. Strategies to prevent antimicrobial resistance in the intensive care unit. Crit Care Med. 2005;33: 1845-53. https://doi. org/10.1097/01.CCM.0000171849.04952.79

16. Ramprasad $B P$, Marissa R, Suprama D. Role of pseudomonas in nosocomial infections and biological characterization of local strains. J Biosci Tech. 2010;11(4): 170-9.

17. Zahra T, Moniri R. Detection of ESBLs and MDR in Pseudomonas Aeruginosa in a Tertiary-Care Teaching Hospital". Iran J Clin Infect Dis. 2011;6(1): 18-23.

18. Pillai J, Yazicioglu C, Moeng S, et al. Prevalence and patterns of infection in critically ill trauma patients admitted to the trauma ICU. South Africa. J Infect Dev Ctries. 2015;9(07): 736-42.

19. Poirel L, Weldhagen G, De Champs C, et al. A nosocomial outbreak of Pseudomonas aeruginosa isolates expressing the extended-spectrum $\beta$-lactamase GES-2 in South Africa J Antimicrob Chemother. 2002 Mar;49(3):561-5. https://doi.org/10.1093/jac/49.3.561

20. Pitout J, Revathi G. L Chow B, al e. Metallo- $\beta$-lactamase-producing Pseudomonas aeruginosa isolated from a large tertiary centre in Kenya. Clin Microbiol Infect. 2008;14(8): 755-9. https://doi. org/10.1111/j.1469-0691.2008.02030.x

21. Koutsogiannou M, Drougka E. Liakopoulos A, al e. Spread of multidrug-resistant Pseudomonas aeruginosa clones in a university hospital. J clin Microbiol. 2013;51(2): 665-8. https://doi.org/10.1128/ JCM.03071-12

22. Harris AD, Jackson SS. Robinson G, al e. Pseudomonas aeruginosa Colonization in the Intensive Care Unit: Prevalence, Risk Factors, and Clinical Outcomes. Infect control Hosp Epidemiol. 2016;37(5): 544548. https://doi.org/10.1017/ice.2015.346

23. Fernández-Barat $L$, Ferrer $M$, De Rosa $F$, et al. Intensive care unitacquired pneumonia due to Pseudomonas aeruginosa with and without multidrug resistance. J Infect. 2016;74(2):142-52.

24. Evans BA, Amyes SGB. OXA $\beta$-lactamases. Clin Microbiol Rev. 2014;27(2): 241-263. https://doi.org/10.1128/CMR.00117-13

25. Poirel L, Weldhagen GF, Naas T, et al. GES-2, a class A $\beta$-lactamase from Pseudomonas aeruginosa with increased hydrolysis of imipenem. Antimicrob Agents. 2001;45 (9):2598-2603. https://doi.org/10.1128/ AAC.45.9.2598-2603.2001

26. Queenan A, Bush K. carbapanemases, the versatile b-lactamases. Clin Microbiol Rev. 2007;20: 440-458. https://doi.org/10.1128/ CMR.00001-07

27. Woodford N, Zhang J, Kaufmann M. E., et al. Detection of Pseudomonas aeruginosa isolates producing VEB-type extended-spectrum betalactamases in the United Kingdom. J Antimicrobial Chemother. 2008;62(6):1265-1268. https://doi.org/10.1093/jac/dkn400

28. Liu We, Liu X, Liao J, et al. Identification of bla OXA-128 and bla OXA-129, two novel OXA-type extended-spectrum- $\beta$-lactamases in Pseudomonas aeruginosa, in Hunan Province, China. J Basic Microbiol. 2010;50(S1):S116-9.. 